\title{
Analysis and Classification Techniques of ECG Signals
}

\author{
Taissir Fekih Romdhane ${ }^{1,2}$, Ridha Ouni ${ }^{3}$, Mohamed Atri ${ }^{4}$ \\ ${ }^{1}$ ENISo, Electrical Engineering Department, University of Sousse, Tunisia \\ ${ }^{2}$ Laboratory of Electronics and Microelectronics, LR99ES30, FSM, Monastir, Tunisia \\ ${ }^{3}$ College of Computer and Information Sciences, Department of Computer Engineering, KSU, KSA \\ ${ }^{4}$ Faculty of Science of Monastir, University of Monastir, Tunisia
}

\begin{abstract}
Due to the gravity of some heart diseases, several researches try to develop robust ECG analysis and classification tools helping physiologists to detect correctly cardiac arrhythmias. In this context, this paper is a good survey of analysis and classification techniques that aims to help physiologic and Data science researchers for a better understanding of different ECG signal processing and classification algorithms. This paper introduces the different ECG signal properties (such as $\mathrm{P}$ wave, R wave, RR interval, PR interval, QRS complex, etc.) and important noises, like base line drift, EMG, muscle contraction, electrode contact noise, etc., that affect strongly this signal. Then, this survey presents various methods and algorithms used to denoise signals collected from MIT-BIH database, to extract features and to classify them into many arrhythmia classes.
\end{abstract}

Keywords: Electrocardiogram; Arrhythmia Detection; Feature extraction; MIT-BIH database;

Signal processing 